\title{
Elastic Beating Pump Using Induced-Charge Electro-osmosis
}

\author{
Hideyuki Sugioka* \\ Department of Mechanical Systems Engineering, \\ Shinshu University, 4-17-1 Wakasato, Nagano 380-8553, Japan
}

(Dated: August 7, 2016)

\begin{abstract}
Pumping a viscous liquid in a confined space is essential in microfluidic systems because the pressure-driven flow rate through small channels decreases with the third or fourth power of the channel size. Hence, inspired by a cilium's pumping ability in a confined space, we propose an elastic beating pump using a hydrodynamic force due to induced-charge electro-osmosis (ICEO) and numerically examine the pumping performance. By the multiphysics coupled simulation technique based on the boundary element method along with the thin double-layer approximation, we find that by selecting the optimum rigidity of the elastic beam, the ICEO elastic beating pump functions effectively at high frequencies with low applied voltages and shows a large average flow velocity with a remarkably large peak velocity that may be useful to flow a liquid with unexpectedly high viscosity. Furthermore, we propose a simple model that explains the characteristics of the time response behavior of the ICEO elastic beating pump to some extent. By this analysis, we can considerably contribute to developments in studies on the artificial cilia having versatile functions.
\end{abstract}

\footnotetext{
* sugioka.hideyuki@canon.co.jp
} 


\section{INTRODUCTION}

Pumping a viscous fluid in a confined space is a challenging problem because the wellknown Hagen-Poiseuille equation tells us that the volume flow rate due to a pressure difference in a circular pipe decreases with not the second power but the fourth power of the radius of the circular pipe. In other words, integrated microfluidic channels lose the ability to pass fluids by a pressure-driven mechanism. Thus, we need to find other fluid transportation mechanisms in microfluidic systems to realize a promising microfluidic system. For this problem, on the one hand, researchers already know that an electro-osmotic flow that uses an electric double layer is one of the solutions because it produces a plug flow that can avoid the limitation of the Hagen-Poiseuille principle. However, the ordinary electro-osmotic flow usually requires a high driving voltage $(\sim 1 \mathrm{kV})$ and the produced flow velocity is relatively low $(\sim 0.1 \mathrm{~mm} / \mathrm{s})$. Consequently, even for a low flow velocity $(\sim 0.1 \mathrm{~mm} / \mathrm{s})$, it requires a large power supply and stultifies miniaturization efforts using a microfluidic chip. On the other hand, it seems that nature has already provided an evolutionary answer to this problem [1-3]. For example, the plant Chara corallina obtained an excellent microscopic beating pump driven by the motor protein myosin at the cell periphery more than 500 million years ago and can transport fluid at $100 \mu \mathrm{m} / \mathrm{s}$ in a confined space under the condition of a low Reynolds number $R e(\equiv \mu R U / D \sim 0.05$ with radius $R=0.5 \mathrm{~mm}$, diffusion constant $D \sim 10^{-9} \mathrm{~mm} / \mathrm{s}$, and $\mu=1 \mathrm{mPa} \mathrm{s}$ ) [4]. Furthermore, the spherical alga Volvox also swims at a high speed (e.g., $200 \mu \mathrm{m} / \mathrm{s}$ ) in a confined space under a low Reynolds number condition (e.g., $R e \sim 0.03$ ) by means of flagella on thousands of surface somatic cells [5]. That is, in fact, nature (in particular, a cilium) has provided a marvelous pumping function along with other useful functions (e.g., mixing and sensing) in a microscale region that suggests innovative design concepts for microfluidic devices. Hence, a biomimic artificial cilium is promising as a key technology that can be applied to a wide range of microfluidic systems, such as a Lab-on-a-chip and micro total analysis systems and it has attracted much attention in this decade [6].

However, the reason why a cilium's motion is useful for transport in a confined space has not been understood completely, although it is recognized that there are three fundamental mechanisms that are active in an a cilia-driven fluid flow, as pointed out by Khaderi et al. [2]: (i) spatially asymmetric motion, (ii) temporally asymmetric motion, and (iii) orienta- 
tionally asymmetric motion. Obviously, the common key point of the three fundamental mechanisms is the symmetry breaking of the beating motion of the elastic beam or filament in a confined space at a small Reynolds number. However, it is still unclear how the symmetry breaking leads to the effective pumping function in a confined space despite the complete symmetry of the Stokes equation for the reverse and forward flows at each time step [7], although the problem has been numerically clarified to some extent $[3,8,9]$. Note that the Stokes equation governs the flow problem under a low Reynolds number condition. Furthermore, it is still unclear how much pumping efficiency it intrinsically has compared with conventional microfluidic pumps, although investigations have been performed extensively for individual problems for a specific motion under a specific condition both experimentally and theoretically. For example, Evans et al. [10] experimentally demonstrated that the motion of cilia-like superparamagnetic rod arrays can be controlled by an externally applied magnetic field. Kim and Netz [3] investigated the pumping efficiency of an array of periodically beating semiflexible filaments by Brownian dynamic simulations. Alexeev et al. [9] simulated the three-dimensional (3D) movement of actuated cilia consisting of elastic filaments using the lattice Boltzmann model for the hydrodynamics and the lattice spring model for the elastics. Nevertheless, the use of biomimetic, artificial cilia is still in its infant stage, as pointed out by Masoud and Alexeev [11], from the viewpoint of engineering applications because artificial cilia are usually driven by large magnetic equipment of at least desktop size [6], which prevents the miniaturization of the microfluidic systems. Obviously, for this problem, electric-driven cilia using metal filaments are useful because they at least remove the problem of large magnetic equipment. In other words, to realize a miniaturized microfluidic system using artificial cilia, it is important to drive the cilia not by magnetic fields but by electric fields. From this context, Toonder et al. [12] experimentally reported that two-dimensional (2D) artificial cilia having a platelike but curled microbeam can be driven by an electric field and can generate a substantial fluid flow $(\sim 0.6 \mathrm{~mm} / \mathrm{s})$ in silicone oil whith an external applied voltage (e.g., $140 \mathrm{~V}$ ). However, the applied voltage of $140 \mathrm{~V}$ is insufficient for realizing a miniaturized microfluidic system because it also requires a large power supply. Furthermore, electric-driven cilia in oil are insufficient for a wide range of biomedical microfluidic applications because biomedical applications usually require pumping functions in aqueous solution, although the analysis is much simpler than that of the electric-driven cilia in water because we need not consider the ion diffusion problem and 
electro-osmotic phenomena. Therefore, the electric-driven cilia using metal filaments in water with small applied voltages should be explored more to realize real innovation, although they have not been explored well, at least theoretically, because of their complexity. That is, the electric-driven cilia in water include the strong coupled phenomena among the ion diffusion, electric migration, fluid flow, and elastic deformation phenomena. Namely, the investigation of the electric-driven (or electrokinetic) cilia requires highly complex multiphysics analysis with new knowledge of the electric double layer around the metal filaments, which has been recognized only recently [13], and thus they have not been investigated extensively yet.

Recently, we have proposed a cilium-like 2D elastic valve using induced-charge electroosmosis (ICEO) and elucidated the design concept as a valve [14]. ICEO [13, 15-17], which includes ac electro-osmosis (ACEO), is caused by the interaction between an electric field and ions in an electric double layer formed by the polarizing effect of the electric field. Thus, it generates a large flow velocity $(\sim 1 \mathrm{~mm} / \mathrm{s})$ proportional to the applied electric field at low applied voltages $(\sim 1 \mathrm{~V})$. Note that conventional linear electro-osmosis only produces a low flow velocity $(\sim 0.1 \mathrm{~mm} / \mathrm{s})$ at high applied voltages $(\sim 1 \mathrm{kV})$, as mentioned before. Consequently, the ICEO phenomenon is promising for microfluidic applications $[13,18]$. Hence, if we can use the hydrodynamic force due to ICEO to drive an artificial cilium having an elastic beam that can beat water periodically, we can realize an electricdriven cilium in aqueous solution with a small voltage and can also realize a miniaturized microfluidic system using innovative artificial cilia because the characteristic of the cilium pump exists in the beating motion of the elastic beam or filament, as mentioned before. Namely, a cilium-like elastic beating pump that uses ICEO has high potential. However, despite its high potential, the elastic beating pump that uses ICEO has not been explored well because of its complexity, as mentioned before; i.e., whether the pump works well or not is unclear, and of course, the liquid pumping mechanism is also unclear.

Therefore, in this study, we focus on a cilia-like 2D elastic beating pump using a hydrodynamic force due to ICEO in water and elucidate its design concept. In other words, our objective in this manuscript is to clarify the liquid pumping mechanism in the presence of an elastic beam driven by the hydrodynamic force due to ICEO. In particular, by the implicit strongly coupled simulation method [14] which solves both fluidic and elastic equations simultaneously by the boundary element method (BEM) along with the thin double-layer 
approximation, we analyze an elastic beating pump using ICEO having two oblique conductive beams that are connected directly to the lower electrode as the minimum artificial cilium. Note that usually the numerical calculation of the interaction problem between a fluid and an elastic is difficult because of the existence of numerical instability, and the reason why we use the implicit strongly coupled simulation method is that it is a markedly stabilized meshless method compared with other methods, as explained in [14]. Namely, through the reliable calculation method, we provide new insight into the liquid pumping mechanism using ICEO. Therefore, our analysis is important. Furthermore, although we often express our elastic beating pump as an artificial pump in this paper according to the convention of the field of artificial cilia [6], it is merely a high-performance elastic beating pump using ICEO, which is not directly relevant to cilia in nature, in the ordinary sense of the field of micro-electro-mechanical systems (MEMS). Therefore, what we focus on is not the relationship between the cilia and our device but the physical pumping mechanism of the ICEO beating pump. In addition, in this manuscript, we first show a simple kinetic analytical theory for the beating motion of an elastic beam due to ICEO and clarify how the symmetry breaking of the ICEO beating pump leads to the effective pumping function in a confined space despite the complete symmetry of the Stokes equation for the reverse and forward flows at each time step [7], although we clarified the static problem of an elastic beam driven by ICEO to realize the valve function in our previous paper [14]. Namely, we present this paper in five sections. That is, through both analytical and numerical kinetic analyses in Sect. 2, we show the analytical and numerical results for the pumping motion of the ICEO beating pump in Sect. 3, and following the discussion in Sect. 4, our conclusions are summarized in Sect. 5 .

\section{THEORY}

\section{A. Structure of the elastic beating pump}

Figure 1 shows a schematic view of an elastic beating pump using ICEO. As shown in Fig. 1(a), we typically place two conductive elastic beams of length $L_{e}=1.5 \mathrm{w}$ and width $d=0.07 w$ on the lower electrode in a rectangular channel of length $2 L=4.5 w$ and width $w=100 \mu \mathrm{m}$ at a $20^{\circ}$ tilt angle $\theta$. Furthermore, Figs. 1(b) and 1(c) show the up- and 


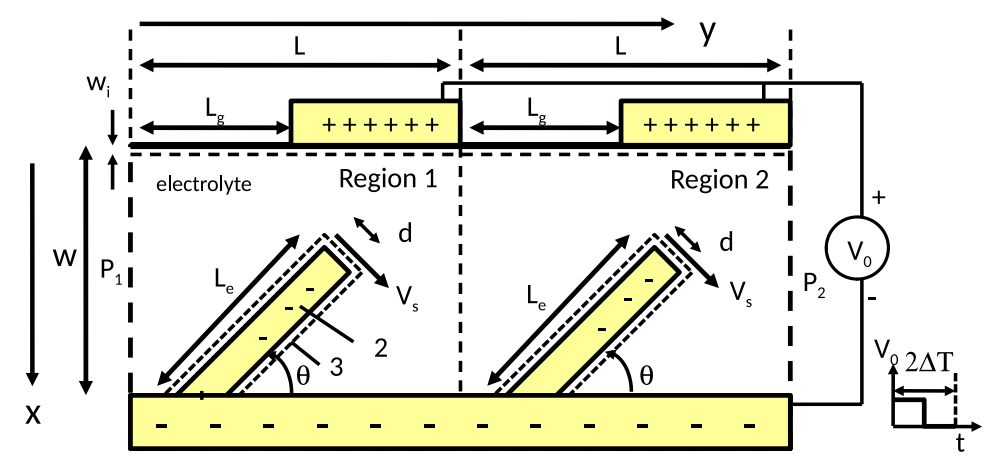

(a) Basic structure of the ICEO beating pump

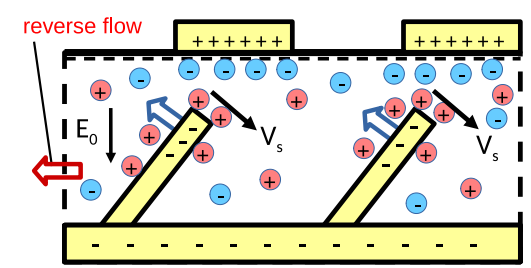

(b) On state (upswing motion)

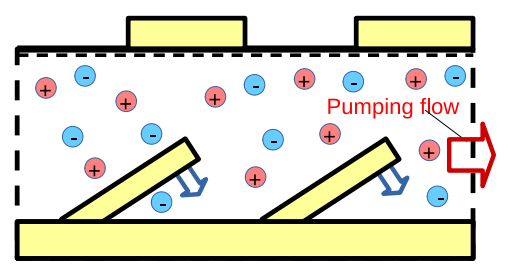

(c) Off state (downswing motion)

FIG. 1. (Color online) Schematic view of an elastic beating pump using induced-charge electroosmosis (ICEO). 1a and 1b: upper and lower electrodes. 2: conductive elastic beam. 3: electro double layer, 4: insulation layer. We place two conductive elastic beams of length $L_{e}=1.5 \mathrm{w}$ and width $d=0.07 w$ on the lower electrode in a rectangular channel of length $2 L=4.5 \mathrm{w}$ and width $w=100 \mu \mathrm{m}$ at a $20^{\circ}$ tilt angle $\theta$. Typically, the applied voltage $V_{0}$ is $1.7 \mathrm{~V}, y_{m}=0.4 w$, the pressure difference $\Delta P\left(\equiv P_{2}-P_{1}\right)$ is $0 \mathrm{~Pa}$, and $P_{1}$ and $P_{2}$ are the pressures at the inlet and outlet, respectively. The Poisson ratio $\nu$ and shear modulus $G$ of the elastic beams are 0.5 and $500 \mathrm{kPa}$, respectively, while the viscosity $\mu$ is $1 \mathrm{mPa}$; furthermore, $V_{s}$ is the slip velocity that is generated by the ICEO phenomenon under the existence of the external electric field $E_{0}=V_{0} / W ; L_{g}=0.5 L$ is the gap length and $w_{1}$ is the thickness of the insulation layer.

downswing motion at the on and off states, respectively. These typical up- and downswing motion of the elastic beams are shown in Fig. 2. As shown in Figs. 2(b) and 2(c), we apply an ac electric voltage of amplitude $V_{0}$ (typically, $1.7 \mathrm{~V}$ ) between the electrodes (1a and 1 b) during $0 \leq t \leq \Delta T$ to move the beam in the upper direction by an ICEO force and switch off the applied voltage during $\Delta T<t \leq 2 \Delta T$ to return the beam to the initial position by an elastic force, where $2 \Delta T\left(=30 T_{0}=30 \mathrm{~ms}\right.$, typically $)$ is the period of the beating of the elastic beam, and $T_{0}=1 \mathrm{~ms}$ and $U_{c}=w / T_{0}=0.1 \mathrm{~m} / \mathrm{s}$ are units for time and velocity, respectively, for convenience, and we set the external pressure difference as 
$\Delta P\left(\equiv P_{2}-P_{1}\right)=0$ throughout this paper. Furthermore, since we assume that the period of the ac voltage is much smaller than $\Delta T$, we can expect the suppression of chemical reactions on the electrodes as a typical merit of an ICEO device. Namely, in our proposed device, the two beams are deflected in the upper direction by the hydrodynamic force due to ICEO during the on-state [in Fig. 2(b)], whereas they return toward the initial position during the off-state [in Fig. 2(c)], without problems caused by chemical reactions. Note that the beams generate an unwanted reverse flow during the on-state [in Fig. 2(b)], whereas they produce a forward pumping flow by the beating effects of the elastic beams during the off-state. Hence, without our analysis it is unclear whether the ICEO beating pump is working. Thus, our analysis is important as a first approach. In particular, there is complete symmetry of the Stokes equation for the reverse and forward flows at each time step [7], as mentioned before. Thus, there is no merit in selecting $\theta \simeq 90^{\circ}$ because of its geometrical symmetry; i.e., to obtain the pumping function in microfluidic channels, we consider the symmetry-breaking structure of the oblique elastic beam of $\theta=20^{\circ}$. Here, we select $\theta=20^{\circ}$ as a first attempt since we can expect the large deflection of the oblique elastic beam structure owing to the ICEO hydrodynamic force, at least for the weak-rigidity beam $(G \simeq 0.01 \mathrm{MPa})$ through our previous analysis of the oblique structure as a valve [14]. Note that obtaining the optimum liquid pumping efficiency of the beating pump is beyond the scope of this study, although it might be important as a future problem. Furthermore, for the sake of practical applications in the future, here we propose the divided structure in the upper electrodes (1a) and show a minimum unit structure that has two oblique conductive elastic beams; i.e., the two beams can be controlled individually in the future. Namely, there is a possibility that the structure will provide another ICEO beating pump that works with a different driving method using the asynchronized motion of two or more beams similar to the pumping movement of natural cilia. However, this is also beyond the scope of this study. Thus, we select the same angle of $\theta=20^{\circ}$ for the two beams and focus on clarifying the pumping mechanism using the synchronized motion of the two elastic beams as a first step. In addition, we also assume an insulation layer of thickness $d^{\text {insulator }}(=0.02 w)$ on the upper electrode to avoid the shortage of the electrical circuit. However, the insulation layer is only modeled numerically as a repulsion force between the upper electrode and the elastic beam, and typically, it does not affect the calculation results because the repulsion force acts only when the beam touches the insulation layer. Furthermore, by using the direct connection 
between the lower electrode and the conductive elastic beam, the typical zeta potential of the edge position of the beam becomes comparable to the applied voltage and thus we can expect a large deflection of the beam, as mentioned in Ref. [14].

\section{B. Multiphysics numerical calculation method for the fluid and elastic objects}

We analyze the elastic beating pump on the basis of BEM along with the thin doublelayer approximation. Here, the thin double-layer approximation is justified because the Debye length is approximately 1000 to $10 \mathrm{~nm}$ for ion concentration of $10^{-7}$ to $10^{-3} \mathrm{M}$ and it is much smaller than the channel width $w$. The multiphysics numerical calculation method is the same as that described in Ref. [14]. However, for the reader's understanding, we briefly explain the method here. Namely, on the basis of the Helmholtz-Smoluchowski formula

$$
\boldsymbol{V}_{s}=-\frac{\epsilon \zeta}{\mu} \boldsymbol{E}_{\mathrm{s}}
$$

we calculate the slip velocity $\boldsymbol{V}_{s}$ on the outside edge of the electric double layer at each time step, where $\boldsymbol{E}_{s}(=-\nabla \phi)$ is the tangential electric field, $\epsilon\left(\sim 80 \epsilon_{0}\right)$ is the dielectric permittivity of the solvent (typically water), $\epsilon_{0}$ is the vacuum permittivity, and $\zeta$ is the zeta potential. Note that since the inside and outside potentials of the electric double layer on the beam $\left(\phi_{i}\right.$ and $\left.\phi_{o}\right)$ are calculated by solving the Laplace equation under the Dirichlet and Neumann boundary conditions, respectively, at each time step, the zeta potentials $\left[\zeta\left(\equiv \phi_{i}-\phi_{o}\right)\right]$ are also calculated at each time step. Therefore, by using the Stokes equations of a fluid

$$
\mu \nabla^{2} \boldsymbol{v}=\nabla p
$$

and Navier's equations of a solid

$$
\nabla \cdot \mu_{e}\left[\nabla \boldsymbol{u}+(\nabla \boldsymbol{u})^{T}\right]+\lambda_{e}(\nabla \cdot \boldsymbol{u}) \boldsymbol{I}=0
$$

with the boundary conditions

$$
\begin{gathered}
\frac{d \boldsymbol{u}}{d t}=\tilde{\boldsymbol{v}}=\boldsymbol{v}-\boldsymbol{V}_{\mathrm{s}}, \\
\boldsymbol{f}_{e}\left(=\boldsymbol{f}_{e}^{\prime}+\boldsymbol{f}^{d e p}\right)=\boldsymbol{f}_{f}
\end{gathered}
$$

on the interface between the fluid and the beams, we can calculate the flow fields and deflections of the beam, where $p$ is the pressure, $\boldsymbol{v}$ is the fluid velocity, $\tilde{\boldsymbol{v}}=d \boldsymbol{u} / d t$ is 
the velocity of the real surface of the beam, $\boldsymbol{u}$ is the displacement, $\mu_{e}$ and $\lambda_{e}$ are the Lame's constants on solid, and $\boldsymbol{f}_{e}$ and $\boldsymbol{f}_{f}$ are the total surface traction vectors (defined by the opposite normal vectors) of the beam and the fluid, respectively. Note that $\boldsymbol{f}_{e}^{\prime}$ is the surface traction due to the deflection of the solid and $\boldsymbol{f}_{e}^{\text {dep }}$ is the surface traction due to

the dielectrophoresis (DEP), and it is described as the Maxwell stress $\boldsymbol{f}^{\mathrm{dep}}=-\frac{1}{2} \epsilon \boldsymbol{E}_{s}^{2} \boldsymbol{n}$, where $\boldsymbol{n}$ is the surface normal unit vector. Furthermore, we use a fixed boundary condition (i.e., $\boldsymbol{u}=d \boldsymbol{u} / d t=0$ ) at the bottom of the elastic beams and we use a constant pressure condition that $P_{1}=P_{2}=0$ at $y=0$ and $4.5 \mathrm{w}$. Furthermore, note that the neglect of the unsteady term in the Stokes equation and the charging time is discussed in detail in [14] with a detailed discussion of the Reynolds number and, consequently, our calculation method is justified.

\section{Simple time response model of the ICEO elastic beating pump}

Because of the nonlinearity of the problem, a complete analytical model of the ICEO elastic beating pump is impossible. However, from the engineering viewpoint, even the simplest model that includes several fitting parameters is useful as the first step. Thus, as mentioned in Sect. 1, here, we show a simple kinetic analytical theory for the beating motion of the elastic beam due to ICEO and clarify how the symmetry breaking of the ICEO beating pump leads to the effective pumping function in a confined space despite the complete symmetry of the Stokes equation for the reverse and forward flows at each time step [7]. By considering the Lorentz reciprocal theorem $[14,19,20]$, we approximate the ICEO force $F^{*, I C E O}$ at the peak beam position $\boldsymbol{x}_{p}$ in the upper direction of the beam in the presence of an electric field as

$$
F^{*, \mathrm{ICEO}} \simeq \mu V_{s}^{e d g e}
$$

where $V_{s}^{\text {edge }}\left(\simeq c_{e} C_{\theta} U_{w}\right)$ is the edge slip velocity, $U_{w}=\epsilon w E_{0}^{2} / \mu$ is the characteristic velocity of ICEO of the channel, $C_{\theta}=\sin 2 \theta / \sin 2 \theta_{0}$ is a factor used to consider the dependence of $V_{s}^{\text {edge }}$ on $\theta$ [13], $\theta_{0}=20^{\circ}$, and $c_{e}$ is a shape factor related to the strength of the local electric field near the peak position $\boldsymbol{x}_{p}$ of the conductive elastic beams. Note that $c_{e}$ is determined as $c_{e} \simeq 3$ from the previous calculation results [14]. By considering linear beam theory, we can approximate the concentrated elastic force $F^{* \text {,elastic }}$ of unit thickness corresponding to 
the deflection $\delta(t)$ of the beam at time $t$ as

$$
F^{* \text { elastic }} \simeq C_{1} \frac{3 E I}{L_{e}^{3}} \delta(t)
$$

where $E=2 G(1+\nu)$ is the Young's modulus, $G$ is the rigidity modulus, $\nu$ is the Poisson ratio, $I=d^{3} / 12$ is the moment of inertia for the rectangular cross section of unit thickness, and $C_{1}(\simeq 0.08)$ is the correction coefficient for the real nonlinear problem. Therefore, by using the same argument as Eq. (6), we obtain the deflection velocity at the peak position $x_{p}$ in an electric field as

$$
\dot{\delta}^{\text {up }}=\frac{C_{2}}{\mu}\left(F^{*, \mathrm{ICEO}}-F^{* \text {,elastic }}\right)
$$

where $C_{2}(\simeq 0.1)$ is also a correction factor for the nonlinear problem and $\delta(t)$ as the deflection value at the peak position. Note that we define the upper direction perpendicular to the elastic beam is positive for the force, velocity, and deflection values. Furthermore, since the phenomenon is complex, we use the superscripts to show the states explicitly; i.e., we use the superscript "up" for the upswing state (on-state) during $0 \leq t \leq \Delta T$, and we use the superscript "down" for the downswing state (off-state) during $\Delta T \leq t \leq \Delta 2 T$. Therefore, by integrating Eq. (8), we obtain

$$
\delta^{\mathrm{up}}(t)=\int_{0}^{t} \dot{\delta}^{\mathrm{up}}\left(t^{\prime}\right) d t^{\prime}(0 \leq t \leq \Delta T)
$$

Furthermore, from Eq. (8), the deflection velocity during downswing motion in the absence of the electric field $\left(F^{*, \mathrm{ICEO}}=0\right)$ is described as

$$
\dot{\delta}^{\text {down }}=-\frac{C_{2}}{\mu} F^{* \text { elastic }}
$$

Therefore,

$$
\delta^{\text {down }}(t)=\int_{0}^{\Delta T} \dot{\delta}^{\text {up }}\left(t^{\prime}\right) d t^{\prime}+\int_{0}^{t} \dot{\delta}^{\text {down }}\left(t^{\prime}\right) d t^{\prime}(\Delta T \leq t \leq 2 \Delta T) .
$$

Since the beam edge position $x_{p}$ is described as

$$
x_{p}=w-L_{e} \sin \theta-\delta^{\text {beam }} \cos \theta,
$$

we can obtain the peak position $x_{p}$ through the simple time response model by the numerical integration of Eqs. (10) and (12). 
We also consider a simple flow velocity model that is related to the pumping efficiency. Firstly, since the volume flow velocities (of unit thickness) due to the up- and downswings of the beam are proportional to the area swept by a beam whose equivalent length is approximated by $\left(L_{e}+\delta\right)$, the spatially averaged flow velocity $U_{p}^{*}$ due to the beating motion of the elastic beam in the $y$-direction is provided as

$$
\begin{array}{r}
U_{p}^{*, \text { up }}=-\frac{1}{2 w}\left(L_{e}+\delta\right) \dot{\delta}^{u p} \tilde{t}^{u p}(0 \leq t \leq \Delta T), \\
U_{p}^{* \text { down }}=-\frac{1}{2 w}\left(L_{e}+\delta\right) \dot{\delta}^{\text {down }} \tilde{t}^{\text {down }}(\Delta T<t \leq 2 \Delta T),
\end{array}
$$

where $\tilde{t}^{\text {up }}$ and $\tilde{t}^{\text {down }}$ are the transmittance factors of the up- and downswings of the beam and are approximated as

$$
\begin{aligned}
\tilde{t}^{\mathrm{up}} & =C_{3}\left(1-x_{p} / w\right), \\
\tilde{t}^{\text {down }} & =\frac{1}{C_{3}}\left(1-x_{p} / w\right),
\end{aligned}
$$

where $C_{3}(\simeq 0.8)$ and $1 / C_{3}(\simeq 1 / 0.8)$ are the proportionality constants for the upward and downward motion, respectively. Note that it is well known that for a low Reynolds number flow governed by the Stokes equation (under the condition that there is no movable part), the flow should be completely reversible and hence the resistance of a channel should be the same for both flow directions, independent of the channel shape, as discussed clearly in [7]. However, for the same reason, if there is a movable part that has spatial asymmetry, the transmittance factors should have reciprocal proportionality constants such as $C_{3}$ and $1 / C_{3}$; in other words, $C_{3}$ is a coefficient concerning the state change from $\mathrm{A}$ to $\mathrm{B}$, and $1 / C_{3}$ is a coefficient concerning the state change from B to A; i.e., to obtain a complete time-reverse flow for the reverse motion of the beam, the above reciprocal relation is required. Note that the above reciprocal relation might not be recognized well because, to the best of our knowledge, we cannot find any clear explanation for the problem in the literature. However, the above assumption (i.e., the reciprocal relation) is needed to at least phenomenologically explain the numerical results for the time response of $U_{p}$ obtained by the BEM. In addition, since the flow is a laminate flow in the channel, the transmittance constants are proportional to $1-x_{p} / w$ as the first approximation.

Secondly, in the presence of the electric field, we need to consider that our proposed pump has a pumping function even for the steady state because of the ICEO slip velocity $V_{s}^{\text {edge }}$ as in other ordinary ICEO pumps $[18,21]$, although the ICEO slip velocity is used to 
move the beam during the motion. Thus, as the first step, we can approximate the spatially averaging flow velocity due to the ICEO slip velocity itself as

$$
U_{p}^{v_{s}}=C_{4}\left(V_{s}^{\text {edge }}-\frac{F^{* \text { up }}}{\mu}\right)(0 \leq t \leq \Delta T),
$$

where $F^{*, u p}=F^{*, I C E O}-F^{* \text { elastic }}$ and $C_{4}(\simeq 0.23)$ is a proportionality constant. Note that $F^{*, \text { up }}$ is balanced with the opposite viscosity force that is produced by the flow velocity $\frac{F^{* \text { up }}}{\mu}$ as in the ordinary Stokes resistance problem. From Eqs. (13), (14), and (17), we obtain a total spatially averaged flow velocity $U_{p}$ that includes both the beating effect and the static slip velocity effect of ICEO; i.e.,

$$
U_{p}=U_{p}^{u p}=U_{p}^{*, u p}+U_{p}^{v_{s}}(\leq t \leq \Delta T), U_{p}=U_{p}^{\text {down }}=U_{p}^{*, \text { down }}(\Delta T<t \leq 2 \Delta T) .
$$

Furthermore, by integrating $U_{p}$ in the time domain, we obtain the pumping liquid volume $V_{p}$ of unit thickness; i.e.,

$$
V_{p}(t)=\int_{0}^{t} U_{p} d t^{\prime}
$$

Thus, we can obtain a time-averaged flow velocity $U_{p, a}$ over the time $2 \Delta T$; i.e.,

$$
U_{p, a}=\frac{1}{2 \Delta T} V_{p}^{t=2 \Delta T}
$$

which indicates the pumping performance of the ICEO elastic beating pump. Note that our model includes an integral expression and we use numerical integration to obtain the specific results of Eqs. (12), (18), (19), and (20) [i.e., $x_{p}(t), U_{p}(t), V_{p}(t)$, and $U_{p, a}$ ], as a first step. However, for simplicity, we call these results the analytical results throughout this manuscript.

Furthermore, in our model, we only consider the motion of one beam in the unit region of length $L$ in Fig. 1. This is because the flow resistance for the beam per unit length $L$ at time $t$ is considered to be equal in Regions 1 and 2 in Fig. 1 for the synchronized motion, as a first step. However, strictly speaking, on the one hand, during the upswing motion, the ICEO flow generated by the the left-hand beam suppresses the upswing motion of the right-hand beam; thus, there is a possibility that the pump efficiency is slightly suppressed by the hydrodynamic interaction. On the other hand, during the downswing motion, the flow generated by the downswing motion of the left-hand beam accelerates the downswing motion of the right-hand beam; thus, there is a possibility that the pump efficiency is slightly 


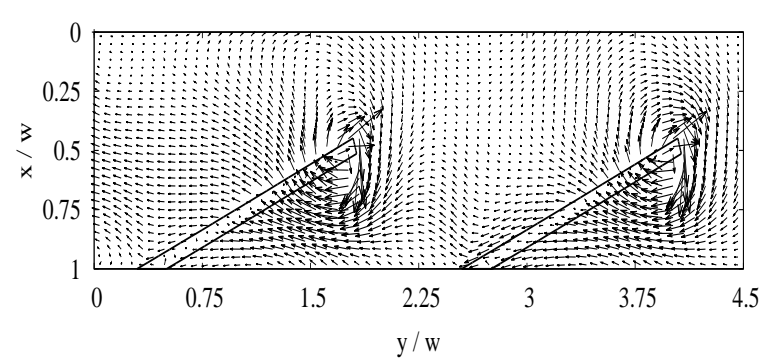

(a) Flow field of upward motion at $t / T_{0}=0$

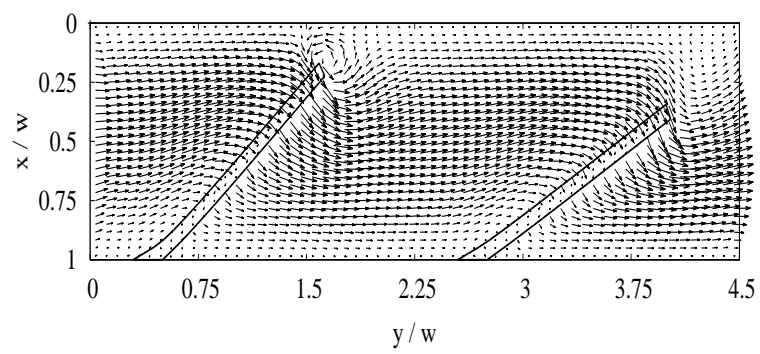

(c) Flow field of downward motion at $t / T_{0}=17$

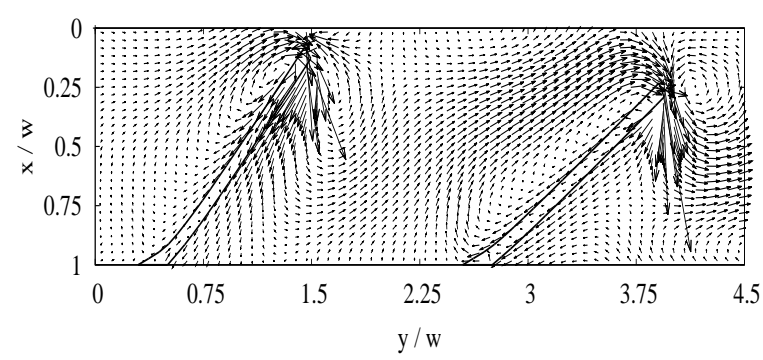

(b) Flow field of downward motion at $t / T_{0}=15$

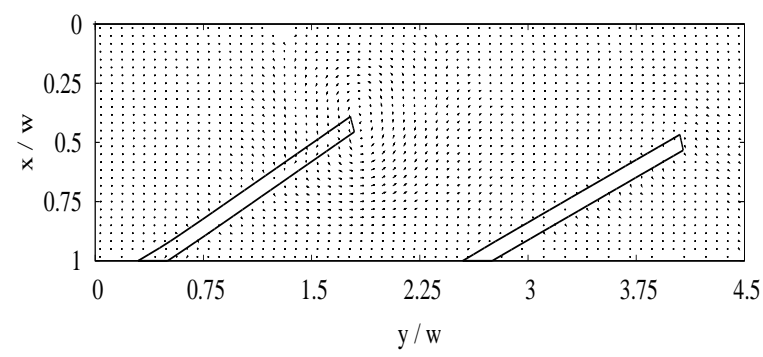

(d) Flow field of downward motion at $t / T_{0}=30$

FIG. 2. Upward and downward motion and flow fields of the ICEO elastic beating pump at $G=200 \mathrm{kPa}$ with $V_{0}=1.7 \mathrm{~V}$. Here, $\nu=0.5, L / w=2.25, L_{e} / w=1.5, d / w=0.07$, and $\theta=20^{\circ}$.

increased by the hydrodynamic interaction. Therefore, we think that the pump efficiency of our model is not significantly changed even if we consider the hydrodynamic interaction effect between beams, although it might be an important problem to consider the asynchronized motion of the beams in the future.

\section{RESULTS}

Figure 2 shows the time evolution of the upward and downward motion and the flow fields of the ICEO elastic beating pump under the conditions $V_{0}=1.7 \mathrm{~V}, 2 \Delta T=30 \mathrm{~ms}, \nu=0.5$, $G=200 \mathrm{k} \mathrm{Pa}, \mu=1 \mathrm{mPa}, L_{e}=1.5 w, d_{e}=0.07 w$, and $\theta=20^{\circ}$. Note that we use $\nu=0.5$, $w=100 \mu \mathrm{m}, T_{0}=1 \mathrm{~ms}, \mu=1 \mathrm{mPa} \mathrm{s}$, and $\Delta P=0 \mathrm{~Pa}$ throughout this manuscript. As shown in Fig. 2(a), initially (at $t / T_{0}=0$ ) because of the ICEO slip velocity at the edge of the beam, the beam edge $x_{p}$ starts to move while generating edge vortices and the upswing motion produces an unwanted backward flow; then we can observe a large deflection at $t=\Delta T=15 T_{0}=15 \mathrm{~ms}$, although the forward pumping flow due to the intrinsic edge slip velocity remains, as shown in Fig. 2(b). Furthermore, Fig. 2(c) shows the downswing motion 
(at $t / T_{0}=17$ ) that beats the fluid strongly. Namely, the downswing motion produces a main forward pumping flow in the $y$-direction and the beam approximately returns to the initial position, as shown in Fig. 2(d). Note that, different from the ICEO elastic valve reported in [14], the ICEO elastic beating pump requires much a larger rigidity and beam thickness than the ICEO elastic valve to beat the fluid strongly and return to the initial position within a short time $(\Delta T=15 \mathrm{~ms})$.

Figure 3 (Fig. 4) shows the time response of the ICEO elastic beating pump at $G=200$, 500 , and $1000 \mathrm{kPa}$ with $V_{0}=1.7 \mathrm{~V}$ (at $V_{0}=1.2,1.5,1.7$, and $2.0 \mathrm{~V}$ with $G=500 \mathrm{kPa}$ ). In Figs. 3 and 4, the characters show the numerical results obtained by the BEM, whereas the lines show the analytical results obtained by the simple time response model [i.e., Eq. (12) for (a), Eq. (18) for (b), and Eq. (19) for (c)], and the numerical results agree fairly well with the analytical results. From Figs. 3 and 4, we find that our simple model clarifies the mechanism of the ICEO elastic beating pump to some extent, although we set $C_{1}=0.08$, $C_{2}=0.1, C_{3}=0.8$, and $C_{4}=0.23$ from our numerical simulations. In particular, as shown in Figs. 3(a) and 4(a), the time response of $x_{p}$ is clearly explained by the force balance among the elastic force $F^{* \text { elastic }}$, the edge ICEO force $F^{*, I C E O}$, and a generalized Stokes resistance force (Stokes drag force). Note that usually the Stokes drag force is defined for a spherical particle; however, a more general flow resistance force and torque that balance with the external force and torque exist [22]. Furthermore, as shown in Figs. 3(b) and 4(b), the mechanism of the ICEO elastic beating pump can be characterized by three kinds of flow velocities; i.e., $U_{p}^{u p-s w i n g}, U_{p}^{v_{s}}$, and $U_{p}^{\text {down-swing }}$ at $t / T_{0}=0,15^{-}$, and $15^{+}$, respectively. Note that it is essential to understand the concept of the force balance between the driving force and the flow resistance in the motion in the low-Reynolds-number world, in which the inertia force can be neglected and physical phenomena are often very different from those in the ordinary experienced world; i.e., the force and torque are always balanced and thus $U_{p}$ can change discontinuously when the force changes discontinuously upon turning off electric fields, as shown in Figs. 4(b) and 4(c). Furthermore, in Fig. 4(b), we find that we obtain the highest average flow velocity of $\sim 7 \mathrm{~mm} / \mathrm{s}$ at $V_{0}=2.0 \mathrm{~V}$. This is an impressive flow velocity for this kind of electro-osmotic pump. Although the Reynolds number also becomes high if the pumping performance becomes high, the maximum Reynolds number Re of our system is $\rho U_{p} w / \mu=0.7<1$, where $\rho\left(=1000 \mathrm{~kg} / \mathrm{m}^{3}\right.$ for water $)$ is the density. Therefore, we can neglect the inertia term even for the worst case. 


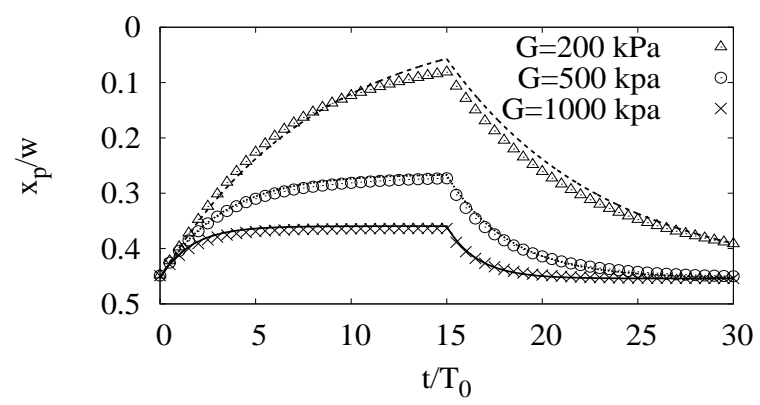

(a) Dependence of $x_{p}$ on time

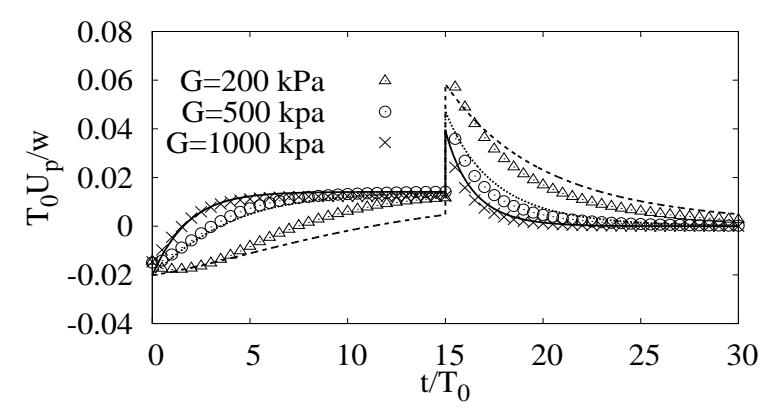

(b) Dependence of $U_{p}$ on time

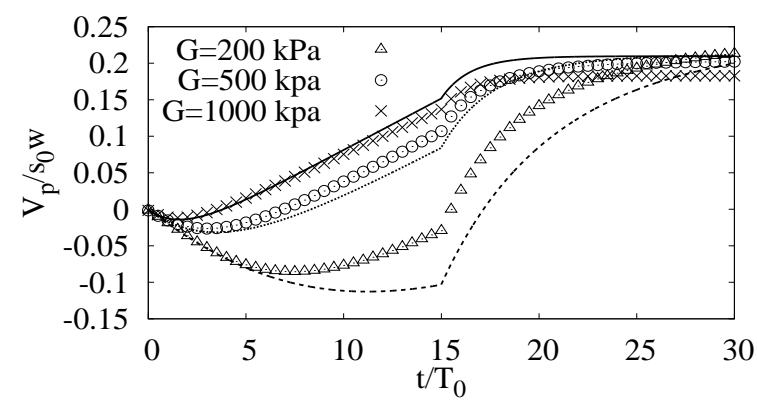

(c) Dependence of $V_{p}$ on time

FIG. 3. Time response of the ICEO elastic beating pump at $G=200,500$, and $1000 \mathrm{kPa}$ with $V_{0}=1.7 \mathrm{~V}$. Here, $L / w=2.25, L_{e} / w=1.5, d / w=0.07$, and $\theta=20^{\circ}$; the dashed, dotted, and solid lines show the analytical results obtained using the simple response model [i.e., Eq. (12) for (a), Eq. (18) for (b), and Eq. (19) for (c)] at $G=200,500$, and $1000 \mathrm{kPa}$, respectively. The triangles, circles, and crosses show the numerical results obtained by the BEM at $G=200,500$, and 1000 $\mathrm{kPa}$, respectively. 


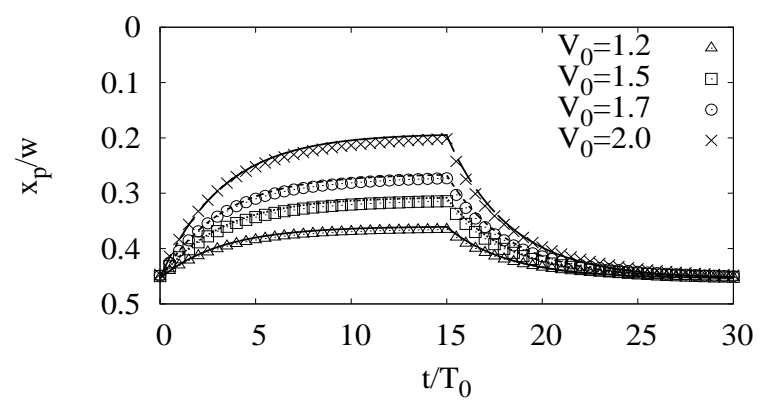

(a) Dependence of $x_{p}$ on time

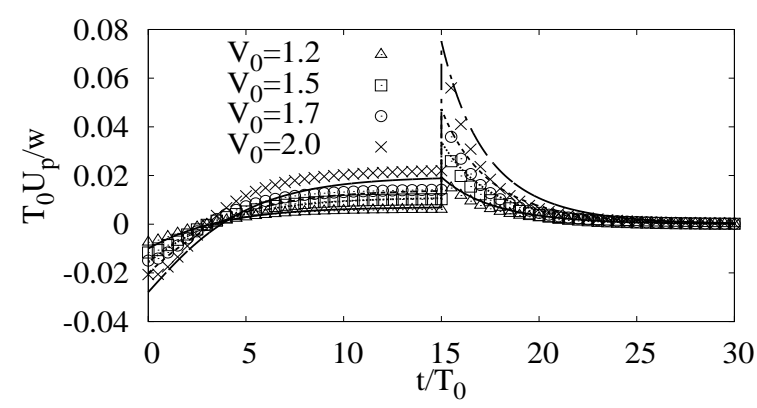

(b) Dependence of $U_{p}$ on time

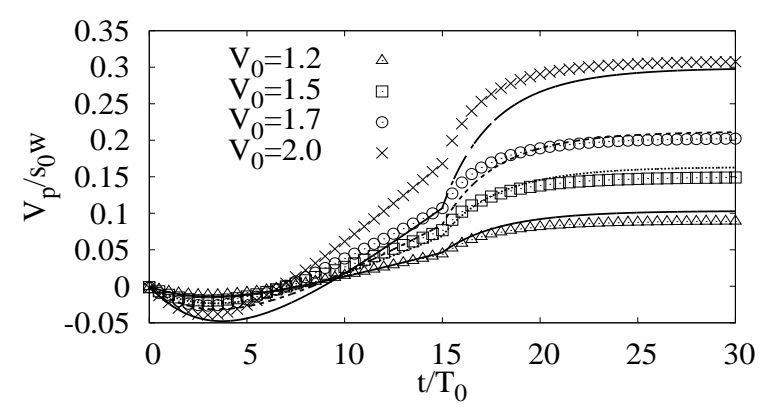

(c) Dependence of $V_{p}$ on time

FIG. 4. Time response of the ICEO elastic beating pump at $V_{0}=1.2,1.5,1.7$, and $2.0 \mathrm{~V}$ with $G=500 \mathrm{kPa}$. Here, $L / w=2.25, L_{e} / w=1.5, d / w=0.07$, and $\theta=20^{\circ}$; the chained, dashed, dotted, and solid lines show the analytical results obtained using the simple response model [i.e., Eq. (12) for (a), Eq. (18) for (b), and Eq. (19) for (c)] at $V_{0}=2.0,1.7,1.5$, and $1.2 \mathrm{~V}$, respectively. The crosses, circles, squares, and triangles show the numerical results obtained by the BEM at $V_{0}=2.0,1.7,1.5$, and $1.2 \mathrm{~V}$, respectively, 


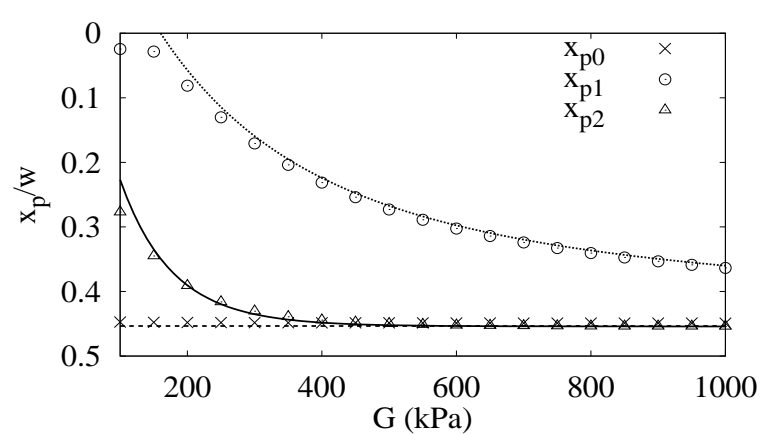

(a) Dependence of $x_{p}$ on $G$

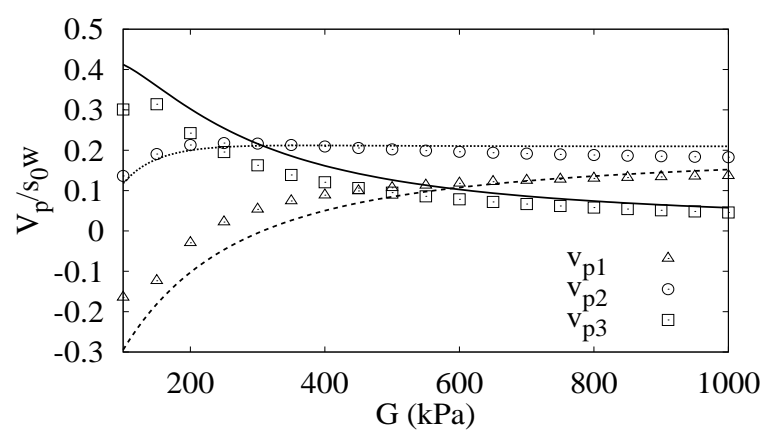

(c) Dependence of $V_{p}$ on $G$

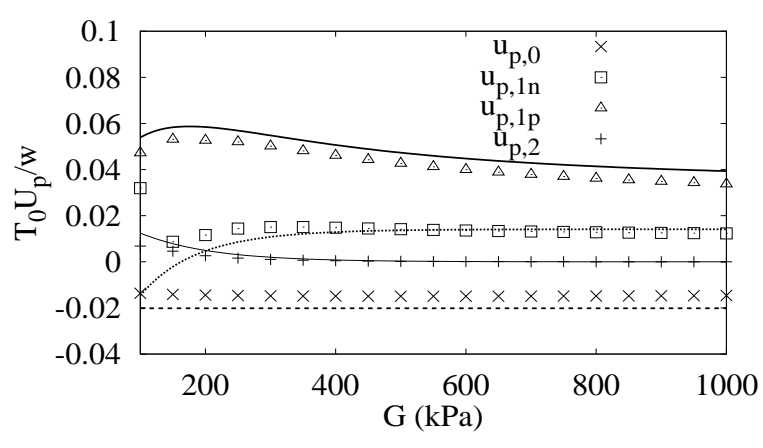

(b) Dependence of $U_{p}$ on $G$

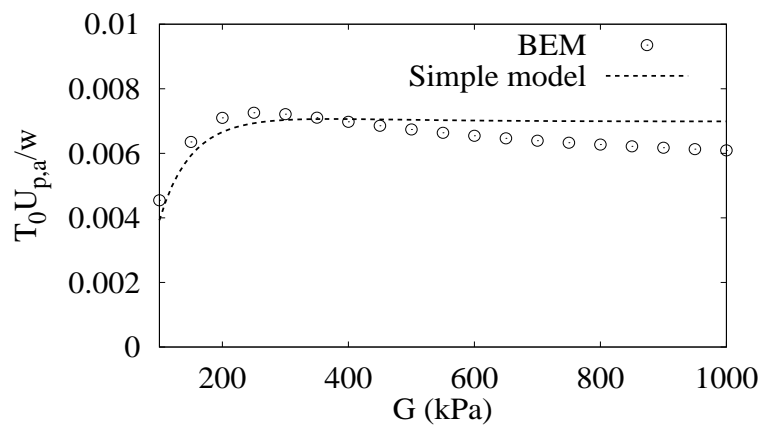

(d) Dependence of $U_{p, a}$ on $G$

FIG. 5. Dependences of $x_{p}, U_{p}, V_{p}$, and $U_{p, a}$ on $G$. Here, $V_{0}=1.7 \mathrm{~V}, L / w=2.25, L_{e} / w=1.5$, $d / w=0.07$, and $\theta=20^{\circ}$; the lines show the analytical results obtained using the simple response model [i.e., Eq. (12) for (a), Eq. (18) for (b), Eq. (19) for (c), and Eq. (20) for (d)], whereas the characters show the numerical results obtained by the BEM; the velocity unit is $U_{c}=w / T_{0}=0.1$ $\mathrm{m} / \mathrm{s}$.

Figures 5 shows the dependences of $x_{p}, U_{p}, V_{p}$, and $U_{p, a}$ on $G$. In Fig. 5 , the lines show the analytical results obtained by the simple response theory [i.e., Eq. (12) for (a), Eq. (18) for (b), Eq. (19) for (c), and Eq. (20) for (d)], whereas the characters show the numerical results obtained by the BEM. Overall, the numerical results agree with the analytical results to some extent, although we may need to develop a more sophisticated model in the future. In Fig. 5(a), the beam edge positions at $t / \Delta T=1$ and $2\left[x_{p 1}\left(=x_{p}^{t=\Delta T}\right)\right.$ and $\left.x_{p 2}\left(=x_{p}^{t=2 \Delta T}\right)\right]$ decrease as the rigidity modulus $G$ increases, and the initial position $\left[x_{p 0}=x_{p}^{t=0}=w-\right.$ $\left.\left.L_{e} \sin \theta\right)\right]$ is constant. These results are reasonable because the deflection of rigid beams should be small and the returning distance of flexible beams in a fixed time should be small. What we want to realize is the beam edge reaching the highest position at $t / \Delta T=1$ and 


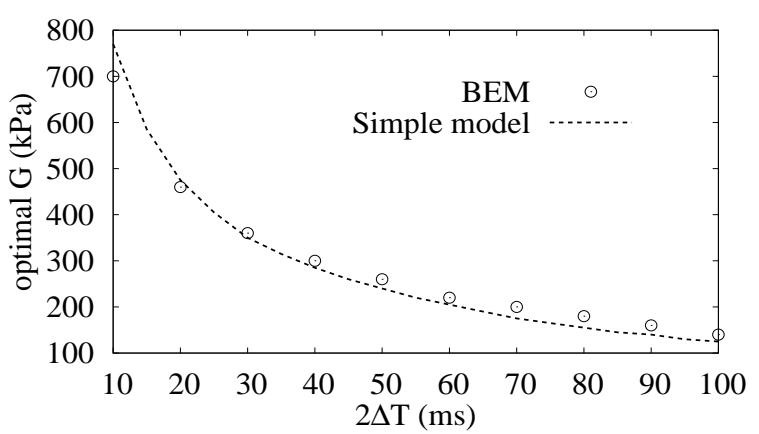

(a) Dependence of $G$ on $2 \Delta T$

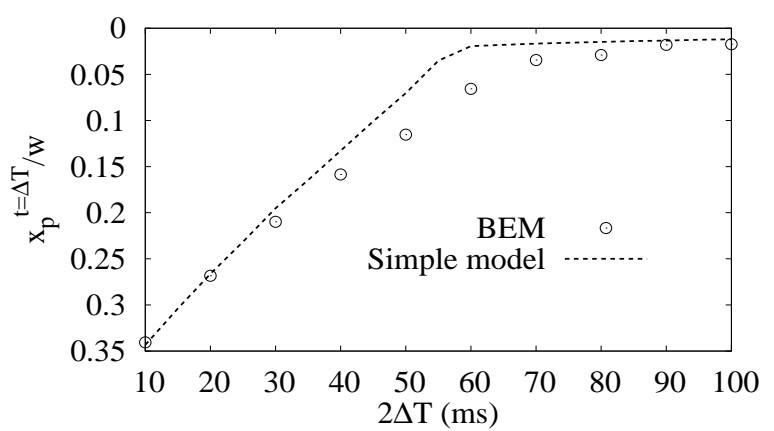

(b) Dependence of $x_{p}^{t=\Delta T}$ on $2 \Delta T$

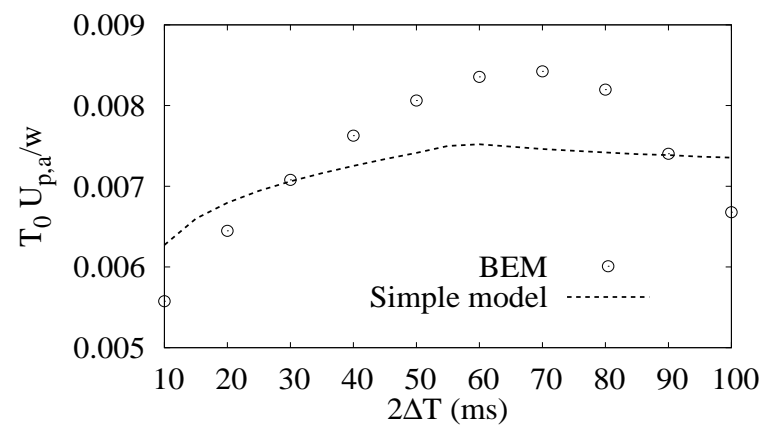

(c) Dependence of $U_{p, a}$ on $2 \Delta T$

FIG. 6. Dependences of $G, U_{p, a}$, and $x_{p}^{t=\Delta T}$ on $2 \Delta T$. Here, $V_{0}=1.7 \mathrm{~V}, L / w=2.25, L_{e} / w=1.5$, $d / w=0.07$, and $\theta=20^{\circ}$; the dashed lines show the analytical results obtained using the simple response model [i.e., Eq. (12) with the condition that $x_{P}^{t=2 \Delta T} \simeq x_{P}^{t=0}$, Eq. (12) for (b); Eq. (20) for (c)] whereas the circles show the numerical results obtained by the BEM; the velocity unit is $U_{c}=w / T_{0}=0.1 \mathrm{~m} / \mathrm{s}$. Note that in (a), $G$ is obtained as the optimum value from Eq. (12) with the condition that $x_{P}^{t=2 \Delta T} \simeq x_{P}^{t=0}$. 
returning to the initial position at $t / \Delta T=2$ for periodic motion. Thus, from Fig. 5(a), we find that $G \simeq 500 \mathrm{kPa}$ is a candidate for the optimum design because $x_{p 2} \simeq x_{p 0}\left(=x_{p}^{t=0}\right)$ at $G \geq 500 \mathrm{kPa}$. In Fig. 5(b), the total average flow velocity $U_{p}$ at $t / \Delta T=1^{-}\left(u_{p, 1 n}=U_{p}^{t=\Delta T^{-}}\right)$ is almost constant at $G \geq 400 \mathrm{kPa}$, whereas $U_{p}$ at $t / \Delta T=1^{+}\left(u_{p, 1 n}=U_{p}^{t=\Delta T^{+}}\right)$slightly decreases in the range $G \geq 200 \mathrm{kPa}$. Here, please recall that, on the one hand, $U_{p}^{t=\Delta T^{-}}$is the average flow velocity for the steady force balance state with the existence of the ICEO torque and it indicates the pump efficiency for the steady mode. Hence, since the average flow velocity is only determined by the value of the ICEO slip velocity $V_{s}^{\text {edge }}$, almost constant value of $T_{0} U_{p}^{t=\Delta T^{-}} / w$ [i.e., $T_{0} U_{p}^{t=\Delta T^{-}} / w \simeq 0.01\left(U_{p}^{t=\Delta T^{-}} \simeq 1 \mathrm{~mm} / \mathrm{s}\right)$ ] is reasonable. On the other hand, since $U_{p}^{t=\Delta T^{+}}$is the average flow velocity for the unsteady force-balanced state between the elastic force and the flow resistance, the decrease is simply explained by the decrease in $x_{p 1}$ [in Fig. 5(a)]. Furthermore, of course, there is no dependence of $u_{p 0}\left(=U_{p}^{t=0}\right)$ on $G$, while $u_{p, 2}\left(=U_{p}^{t=2 \Delta T}\right)$ slightly decreases as $G$ increases, corresponding to the decrease in $\left|x_{p 2}-x_{p 0}\right|$ [in Fig. 5(a)]. In Fig. 5(c), $v_{p 1}\left(=V_{p}^{t=\Delta T}\right)$ is the total pumping volume during the upward motion of the beam, $v_{p 2}\left(=V_{p}^{t=2 \Delta T}\right)$ is the total pumping volume at $t / \Delta T=2$ (i.e., the net pumping volume of one cycle), $v_{p 3}\left(=v_{p 2}-v_{p 1}\right)$ is the pumping volume during the downward motion of the beam, and $s_{0}$ is the area of the cross section of the channel. Here, what we want to determine is the condition for the maximum of $v_{p 2}$ when $G \geq 500$ $\mathrm{kPa}$. Since $v_{p 2}$ decreases slightly as $G$ increases, as shown in Fig. $5(\mathrm{~b})$, we find that $G \simeq 500$ $\mathrm{kPa}$ is still the candidate for the optimum design. In Fig. $5(\mathrm{~d}), U_{p, a}$, which indicates the average pumping performance of the ICEO elastic beating pump, has the maximum value $\left(U_{p, a}^{\max }=7.3 \times 10^{-3} w / T_{0}=0.73 \mathrm{~mm} / \mathrm{s}\right)$ at $G=250 \mathrm{kPa}$. This is because as $G$ increases, the maximum deflection $\delta^{t=\Delta T}$ [corresponding to $x_{p 1}$ in Fig. $5(\mathrm{a})$ ] and the pumping volume in the downswing motion $\left[v_{p 3}\right.$ in Fig. $\left.5(\mathrm{c})\right]$ decrease, whereas as $G$ decreases, the backward pumping volume in the upswing motion $\left[v_{p 1}\right.$ in Fig. $\left.5(\mathrm{c})\right]$ increases. Furthermore, rigorously, the final edge position $x_{p 2}$ does not agree completely with the initial edge position $x_{p 0}$ at $G<500$ KPa. Thus, to realize ideal cyclic beating, $G \geq 500 \mathrm{kPa}$ is required. Here, by considering the previous argument, $G=500 \mathrm{kPa}$ is the optimized value for the period $2 \Delta T=30 \mathrm{~ms}$ [as is also expected from Figs. 5(a)-5(c)].

Figures $6(\mathrm{a})-6(\mathrm{c})$ show the dependences of the optimum rigidity $G^{o p t}$, the edge position $x_{p}^{t=\Delta T}$, and the optimum time-averaged velocity $U_{p, a}^{o p t}$ on $2 \Delta T$ under the conditions $V_{0}=1.7$ $\mathrm{V}, L / w=2.25, L_{e} / w=1.5, d / w=0.07, \nu=0.5, w=100 \mu \mathrm{m}, T_{0}=1 \mathrm{~ms}, \mu=1 \mathrm{mPa}$ 
s, $\theta=20^{\circ}$, and $\Delta P=0$ Pa. As shown in Fig. 6(a), $G^{\text {opt }}$ decreases as $2 \Delta T$ increases. Thus, as $2 \Delta T$ increases, $x_{p}^{t=\Delta T}$ approaches the upper electrode (at $x=0$ ) and maintains its position from $2 \Delta T \simeq 70 \mathrm{~ms}$, as shown in Fig. 6(b). Therefore, we can observe the peak value $U_{p, a}^{\text {peak }}(2 \Delta)(=0.84 \mathrm{~mm} / \mathrm{s})$ at $2 \Delta T \simeq 70 \mathrm{~ms}$ (i.e., beating frequency $f=14.3 \mathrm{~Hz}$ ) and $G=200 \mathrm{kPa}$, as shown in Fig. 6(c). In other words, the strategy of a slower beating frequency with a larger deflection is superior to the strategy of a faster beating frequency with a smaller deflection until the limit of the maximum deflection because of the channel width $w$. Furthermore, in Fig. 6, the dashed lines show the analytical results obtained using the simple response model [i.e., Eq. (12) with the condition that $x_{P}^{t=2 \Delta T} \simeq x_{P}^{t=0}$, Eq. (12) for (b); Eq. (20) for (c)], whereas the circles show the numerical results obtained by the BEM. As shown in Fig. 6, the simple model explains the fundamental design concept

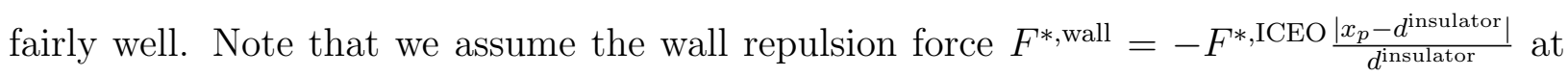
$\left|x_{p}\right| \leq d^{\text {insulator }}(=0.02 w)$ even for the simple model because the limitation of the deflection due to the channel wall intrinsically provides the optimum beating period at $2 \Delta T \simeq 70 \mathrm{~ms}$.

\section{DISCUSSION}

Although an ICEO elastic valve was proposed in a previous paper [14], a cilia-like ICEO elastic beating pump was first proposed here and the design concept was clarified in this manuscript. That is, different from the ICEO elastic valve [14], the ICEO elastic beating pump requires a much higher rigidity to push the fluid strongly and to return to the initial position within a beating period. The calculation results show that the average flow velocity in a beating period is larger $(\sim 0.7 \mathrm{~mm} / \mathrm{s})$ than that of an ordinary de electro-osmotic pump $(\sim 0.1 \mathrm{~mm} / \mathrm{s})$ and it is similar to that of the highest-speed electro-osmotic pumps $(\sim 1$ $\mathrm{mm} / \mathrm{s}$ ) [18]. Note that the highest-speed pump was obtained using a coplanar asymmetrical electrode structure having a step-shaped electrode on one side [18] and the pump should be classified as an ICEO pump in the general sense [13], although the ICEO phenomenon of the electrodes is called ac electro-osmosis (ACEO) [15]. Furthermore, the ICEO elastic beating pump has a large peak velocity $\left(\sim 7 \mathrm{~mm} / \mathrm{s}\right.$ at $\left.V_{0}=2.0 \mathrm{~V}\right)$ that may be useful to strongly flow a fluid with an unexpectedly high viscosity in a real application and probably contributes to making a system robust. In addition, the ICEO elastic beating pump has various possibilities; e.g., (i) there is a possibility that even an unexpected solid obstacle can 
be transported by the mechanical beating motion of the beams; (ii) the beating motion can contribute to the self-cleaning function of an active surface and it can be a key technology because ICEO is sensitive to contamination of the surface [23]; (iii) the motion of the oblique elastic beam can be used to realize other indispensable functions, such as valves, mixers, and sensors, with almost the same structure. Hence, the ICEO elastic beating pump is important, although we need to clarify its various possibilities in the future.

\section{CONCLUSIONS}

We have proposed an elastic beating pump using induced charge electro-osmosis around a conductive elastic beam in water and numerically examined the large pumping performance. By an implicit strongly coupled calculation method between a fluid and an elastic structure along with a simple analytical time response model, we find that (1) the optimum average flow velocity in one beating cycle is approximately $0.7 \mathrm{~mm} / \mathrm{s}$ and the maximum flow velocity caused by the downswing of the beam is approximately $7 \mathrm{~mm} / \mathrm{s}$. (2) The slower beating strategy provides better pumping performance than the faster beating strategy until the limit of the deflection due to the channel width. (3) The ICEO elastic beating pump intrinsically requires higher rigidity than the ICEO elastic valve because the main forward flow is generated from the strong elastic downswing within the beating period. (4) The ICEO elastic beating pump is characterized by the unwanted backward flow velocity due to the upswing motion of the beam, the main forward flow velocity due to the downswing motion of the beam, and the intrinsic ICEO slip flow velocity at the edge position. In particular, we find that by accumulating the ICEO flow energy as an elastic energy, the performance of liquid pumping in a narrow channel is improved considerably, at least for an instant velocity, by the beating of elastic beams compared with that of ordinary ICEO pumps, which are considered to be state-of-the-art microfluidic pumps using an electro-osmotic phenomenon; i.e., the pumping performance of the ICEO elastic beating pump is the highest ever reported, at least theoretically, for this kind of pump and we succeeded in clarifying its mechanism. 


\section{ACKNOWLEDGMENTS}

This work was partially supported by JSPS KAKENHI Grant Number JP16K05650.

[1] A. Shields, B. Fiser, B. Evans, M. Falvo, S. Washburn, and R. Superfine, PNAS 107, 15670 (2010).

[2] S. N. Khaderi, M. G. H. M. Baltussen, P. D. Anderson, J. M. J. den Toonder, and P. R. Onck, Phys. Rev. E 82, 027302 (2010).

[3] Y. W. Kim and R. R. Netz, Phys. Rev. Lett. 96, 158101 (2006).

[4] J.-W. van de Meent, I. Tuval, and R. E. Goldstein, Phys. Rev. Lett. 101, 178102 (2008).

[5] K. Drescher, K. C. Leptos, I. Tuval, T. Ishikawa, T. J. Pedley, and R. E. Goldstein, Phys. Rev. Lett. 102, 168101 (2009).

[6] J. den Toonder and P. Onck, Trends Biotechnol. 31, 85 (2013).

[7] A. Groisman and S. R. Quake, Phys. Rev. Lett. 92, 094501 (2004).

[8] S. Camalet, F. Jülicher, and J. Prost, Phys. Rev. Lett. 82, 1590 (1999).

[9] A. Alexeev, J. M. Yeomans, and A. C. Balazs, Langmuir 24, 12102 (2008).

[10] B. Evans, A. Shields, R. Carroll, S. Washburn, M. Falvo, and R. Superfine, Nano Lett. 7, $1428(2009)$.

[11] H. Masoud and A. Alexeev, Soft Matter 7, 8702 (2011).

[12] J. d. Toonder, F. Bos, D. Broer, L. Filippini, M. Gillies, J. de Goede, T. Mol, M. Reijme, W. Talen, H. Wilderbeek, V. Khatavkar, and P. Anderson, Lab Chip 8, 533 (2008).

[13] M. Z. Bazant and T. M. Squires, Phys. Rev. Lett. 92, 066101 (2004).

[14] H. Sugioka, Phys. Rev. Appl. 3, 064001 (2015).

[15] A. Ramos, A. Gonzalez, A. Castellanos, N. G. Green, and H. Morgan, Phys. Rev. E 67, $056302(2003)$.

[16] M. Z. Bazant, M. S. Kilic, B. D. Storey, and A. Ajdari, Adv. Colloid Interface Sci. 152, 48 (2009).

[17] F. Ziebert, M. Z. Bazant, and D. Lacoste, Phys. Rev. E 81, 031912 (2010).

[18] J. P. Urbanski, T. Thorsen, J. A. Levian, and M. Z. Bazant, Appl. Phys. Lett. 89, 143508 (2006). 
[19] H. Sugioka, Colloids and Surfaces A: Physicochem. Eng. Aspects 376, 102 (2011).

[20] M. C. Fair and J. L. Anderson, J. Colloid Interface Sci. 127, 388 (1989).

[21] H. Sugioka, Phys. Rev. E 78, 057301 (2008).

[22] H. Brenner, Chem. Eng. Sci. 19, 703 (1964).

[23] R. Messinger and T. Squires, Phys. Rev. Lett. 105, 144503 (2010). 